\title{
Hunting for huntingtin modification
}

Researchers from the University of British Columbia in Vancouver have gained new insight into the pathogenesis of Huntington's disease (HD). Their study shows that a particular modification to the huntingtin (HTT) protein is essential for its normal trafficking and function, and becomes disrupted in mutant HTT.

The progressive cognitive and motor decline that occurs in HD is associated, at the cellular level, with the formation of nuclear and cytoplasmic inclusions of aggregated HTT protein - caused by mutations that expand the polyglutamine (polyQ) tract of the protein. It was thought that these inclusions might contribute to the neuronal toxicity and eventual apoptosis that characterizes the pathogenesis of HD. However, mounting evidence suggests that they might instead be a marker rather than a cause of the disease. For example, in a mouse model of the disease (YAC128), the occurrence of inclusions is preceded by the occurrence of motor and cognitive decline. By contrast, in another mouse strain that expresses just a short portion of HTT protein, inclusions develop but the neuronal dysfunction and degeneration seen in YAC128 mice do not. So what does lead to toxicity and cell death in HD?

Palmitoylation is a recently identified post-translational protein modification that has been shown to regulate the trafficking and function
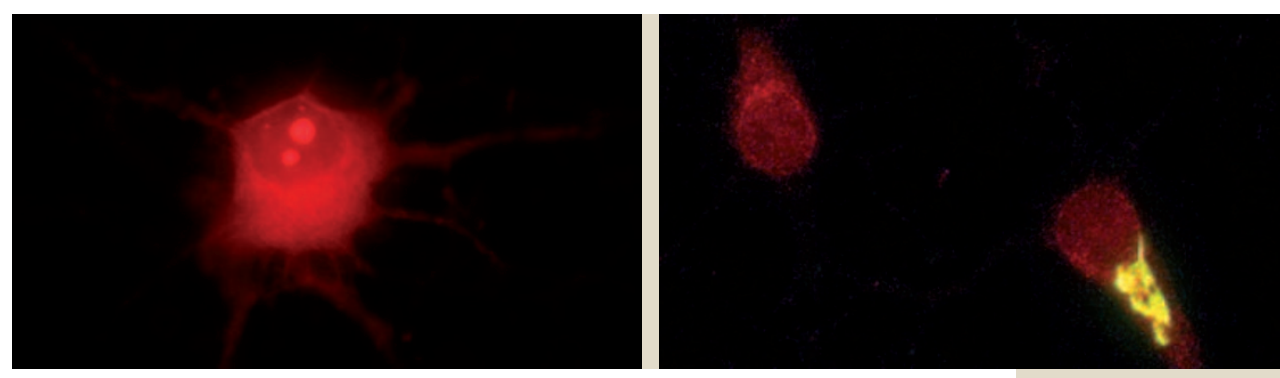

of a number of membrane-associated proteins. Because normal HTT protein localizes to plasma and intracellular membranes, and has been shown to be palmitoylated (by huntingtin interacting protein, HIP14), the team considered that palmitoylation might also regulate HTT's normal function. And it does.

The team first identified which residue of HTT gets palmitoylated. Then, by preventing palmitoylation of both wild-type and mutant HTT, the team observed an increased formation of inclusions (not normally seen for the wild-type protein), indicating that the distribution of HTT was disrupted and suggesting a role for palmitoylation in normal trafficking and folding of HTT.

The authors identified that the amount of palmitoylation of HTT is significantly reduced in the presence of the expanded polyQ tract, suggesting that the expansion might interfere with palmitoylation of HTT by HIP14. To investigate whether reduced palmitoylation could contribute to neuronal toxicity in $\mathrm{HD}$, the team measured the degree of apoptosis in cells expressing either wild-type or mutant HTT when exposed to NMDA ( $N$-methyl-Daspartate)-induced toxicity. Cells expressing mutant HTT showed increased apoptosis in response to NMDA compared with those expressing wild-type HTT, but importantly both cell types became significantly more susceptible when palmitoylation of the proteins was abolished.

The findings by Yanai and colleagues suggest that without correct palmitoylation the normal cellular distribution of HTT gets disturbed and, as a result, the protein becomes cytotoxic. Encouragingly, however, the team found that overexpression of HIP14 considerably reduced the number of inclusions in cells containing mutant HTT. Therefore, discovering how palmitoylation of mutant HTT might be increased (or depalmitoylation prevented) could lead to drugs with the potential to slow or even stop the progressive neurodegeneration in patients with HD.

Ruth Williams
Neurons expressing palmitoylation-resistant mutant HTT form nuclear inclusions (right), increased expression of HIP14 influences norma distribution of HTT (left) Image courtesy of A. Yanai and colleagues, University of British Columbia, Canada.

ORIGINAL RESEARCH PAPER Yanai, A. et al. Palmitoylation of huntingtin by HIP14 is essential for its trafficking and function. Nature Neuro. 21 April 2006 (doi:10.1038/ $\mathrm{nn1702)}$

\section{RESEARCH HIGHLIGHTS ADVISORS}

NANCY ANDREASEN

University of lowa, IA, USA ALLAN BASBAUM

University of California San Francisco, CA, USA

RANDY BUCKNER

Washington University MO, USA
DAVID CLAPHAM

Harvard Medical School, MA, USA

PIETRO DE CAMILLI

Yale University School of Medicine,

CT, USA

BARRY EVERITT

University of Cambridge, UK
GORDON FISHELL

Skirball Institute, NY, USA ENNEDY California Institute of Technology, CA, USA LYNN NADEL

University of Arizona, AZ, USA

DENNIS O'LEARY

The Salk Institute, CA, USA
TERRY SEJNOWSK

The Salk Institute, CA, USA WOLF SINGER Max-Planck-Institut für Hirnforschung, Germany CLAUDIO STERN

University College London, UK
PATRICK TAM

Children's Medical Research Institute. Sydney, Australia RICHARD W. TSIEN

Stanford University School of

Medicine, CA, USA

RAFAEL YUSTE

Columbia University, NY, USA 


\section{In the news}

\author{
MIND CONTROL
}

The stress of having to write this article in just 1 hour makes it easy to focus the mind. However, in normal daily life there is a constant barrage of distractions to contend with. For people with attention deficit and hyperactivity disorder (ADHD), filtering out these unwanted distractions and concentrating on the task in hand is no easy matter. ADHD sufferers essentially "pay attention to too many things" says Candice Drouin, University of Philadelphia (New Scientist, 3 June 2006).

The drug Ritalin has been used to treat ADHD patients for more than two decades. However, despite its common use, little was known about how this amphetamine relative actually worked. Now, Drouin and her colleagues report in the Journal of Neurophysiology that, in rats, Ritalin raises the level of the neurotransmitter noradrenaline and simultaneously suppresses the brain's response to extraneous sensory input. "It probably helps the animal to focus on what's new and not be distracted by what's familiar" says Druoin (New Scientist, 3 June 2006).

The team measured the activity of neurons of the somatosensory cortex of rats while stimulating their whiskers. They found that in rats that were given a low or moderate dose of methylphenidate (Ritalin) the level of noradrenaline was higher, and also that the long-latency phase of the brain's response to sensory stimuli was reduced. This is believed to help filter out the sensory noise (Medical News Today, 31 May 2006).

So how does this study relate to ADHD in humans? Barry Waterhouse, who co-authored the paper, says "this experiment adds to our knowledge of what the drug is doing at the cellular level" and suggests that the question for future studies is "how does the individual's perception of what is an important stimulus factor into the equation?" (Medical News Today, 31 May 2006).

Ruth Williams

\section{DEVELOPMENT}

Instructions for induction

Neural crest cells are stem cells that appear along the border of the neural plate early in development, destined to differentiate into a wide variety of cell types throughout the body. Because interactions between neural and non-neural ectoderm and/or mesoderm were thought to be important for neural crest induction, it was previously assumed that induction occurred after neural plate formation (stage 5). Contrary to this, in new work reported in Nature, Basch et al. show that early neural crest induction occurs at or before gastrulation (stage 3 ), and requires the transcription factor PAX7.

To investigate neural crest induction, the authors looked at the expression of known neural crest markers at progressively earlier stages and identified a region of $\mathrm{PAX} 7$ expression - first appearing with the definitive

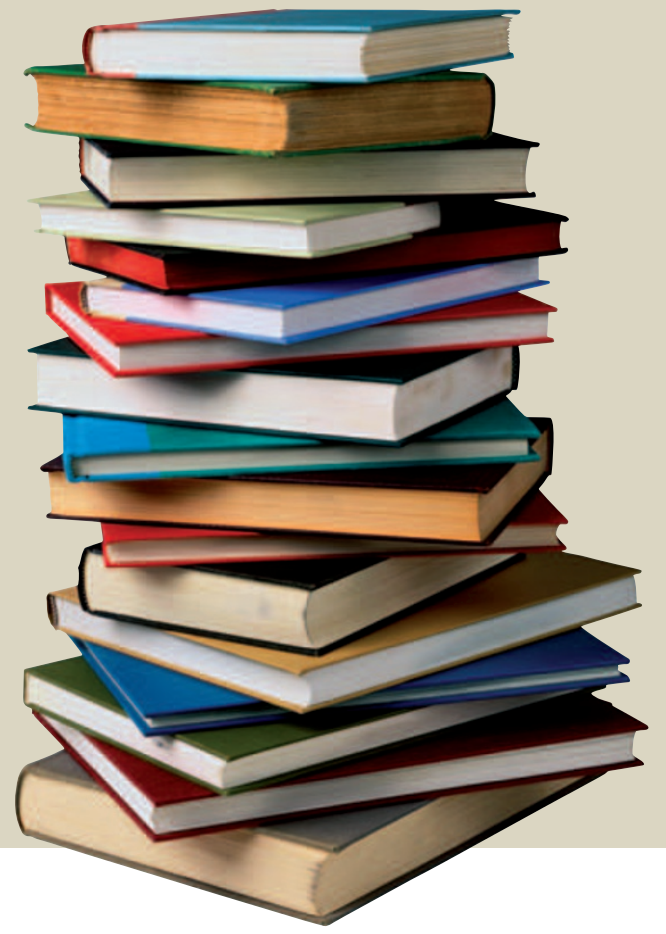

PSYCHIATRIC DISORDERS

\section{Homing in on the target of antidepressants}

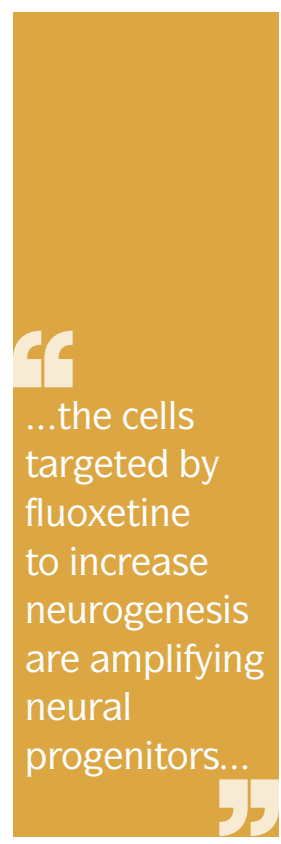

The mechanism of action of most commonly used antidepressants is poorly defined and a better understanding of the effects of these drugs on the brain could lead to new therapeutic approaches. A potential link between neurogenesis and antidepressant treatment announced several years ago generated excitement in the field, but the mechanisms underlying this connection have remained elusive. Now a new study by Enikolopov and colleagues narrows down the target of at least one class of antidepressants to a specific population of early progenitor cells.

Neurons are continuously generated in the dentate gyrus region of the hippocampus throughout life. In animal models, this activity increases in response to various types of antidepressant therapy, including selective serotonin reuptake inhibitors (SSRIs) such as fluoxetine, and potentially contributes to their therapeutic effects. However, the precise stage of the multi-step process of neurogenesis at which SSRIs actually intervene is unknown. A clearer understanding of the progenitor cell type targeted by these drugs could enhance understanding of the pathogenesis of depression and the development of improved antidepressants.

Quantification and characterization of different progenitor cell types by immunofluorescence is difficult, owing to the widespread, cytoplasmic expression of typical marker proteins such as nestin, combined with dense packing of the cells within the dentate gyrus. The authors therefore generated mice in which a fluorescent signal localized specifically to the nucleus of these cells, producing a labelling pattern that was easier to quantify. In combination with staining for various proteins that are expressed at different stages of neurogenesis, the authors defined six distinct sequential stages 
primitive streak (stage $4+$ ) - that correlated with the putative neural crest domain. They then looked even earlier, at bilaminar chick embryos undergoing gastrulation (stage 3/4). Using a fluorescent lipophilic probe, they traced the fate of cells in the prospective PAX7 domain, prior to expression of PAX7. Cells from this region were incorporated into dorsal neural folds and, later, into the migrating neural crest cell population.

To determine whether cells within the prospective $\mathrm{PAX} 7$ expression domain have the ability to form neural crest cells in the absence of exogenous signals, Basch and colleagues explanted onto collagen gels several segments of a strip of epiblast - the outer layer of a blastula that gives rise to the ectoderm after gastrulation. Medial segments - between the primitive streak and the area opaca/area pellucida border - were able to generate migratory neural crest cells. No neural, node or mesodermal markers were found in these explants, showing that they were already specified to form neural crest cells, and contradicting the previous belief that interaction between tissue layers is required for neural crest induction.

Antisense oligonucleotides were then used to block the translation of Pax7 mRNAs to investigate the role of PAX7 in vivo. When these were electroporated into the presumptive neural crest domain of stage 4 embryos, there was a marked decrease in PAX7 protein levels and expression of neural crest markers compared with the contralateral side, confirming the necessity of PAX7.

These findings reveal an important role for PAX7 in neural crest formation, which is induced earlier than was previously thought and can occur independently of mesoderm and neural tissues. Preliminary findings in the same laboratory suggest that this mechanism of neural crest induction might be conserved in all amniotes.

\section{Daniel McGowan}

ORIGINAL RESEARCH PAPER Basch, M. L. et al. Specification of the neural crest occurs during gastrulation and requires Pax7. Nature 441, 218-222 (2006) of neuronal development and investigated the effects of chronic fluoxetine treatment on the proliferative activity of cells at each stage. Using this approach, the authors showed that the cells targeted by fluoxetine to increase neurogenesis are amplifying neural progenitors - the cells generated in the second 'stage' of neurogenesis as defined in this study.

It will be vital to determine whether these cells are a common target for other types of antidepressant, and it remains to be confirmed whether these effects will translate to the human condition. Nevertheless, this study sheds light on the cells targeted by SSRIs in the brain and should direct further studies to define the precise molecular targets of these drugs. Furthermore, this model allows different early progenitor types to be more clearly defined, which could contribute to a better understanding of hippocampal neurogenesis and the pathological effects of depression.

Katherine Whalley, Associate Editor, Nature Reviews Drug Discovery

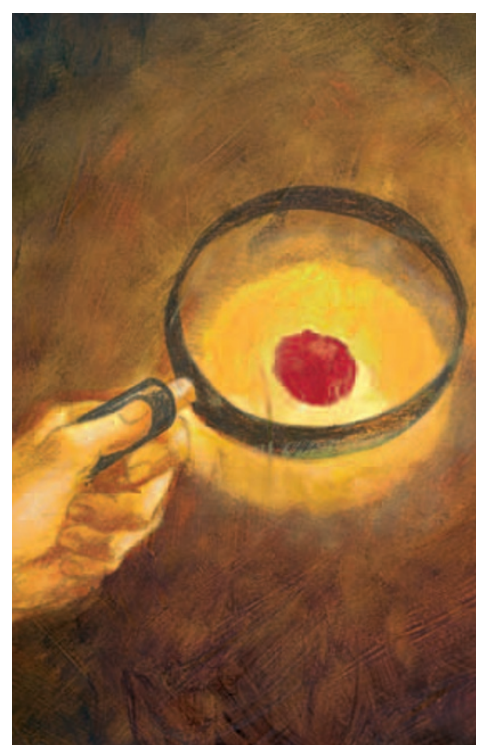

ORIGINAL RESEARCH PAPER Encinas, J. M et al. Fluoxetine targets early progenitor cells in the adult brain. Proc. Natl Acad. Sci. USA 103 , 8233-8238 (2006)

FURTHER READING Duman, R. S. et al. Regulation of adult neurogenesis by antidepressant treatment.

Neuropsychopharmacology 25, 836-844 (2001)| Santarelli, L. et al. Requirement of hippocampal neurogenesis for the behavioural effects of antidepressants. Science 301, 805-809 (2003)

\section{$\Rightarrow$ DEVELOPMENT}

\section{The triple-action factor}

\section{The development of post-mitotic neurons from neural} precursors involves three steps: cell cycle exit, cell migration and cell differentiation. The cyclin-dependent kinase inhibitor $\mathrm{p} 27^{\mathrm{kip} 1}$ is known to be involved in controlling cell cycle exit, but now a new study by Guillemot and colleagues shows that it also regulates both migration and differentiation. This multitalented protein performs each step by a different mechanism.

In the cerebral cortex of

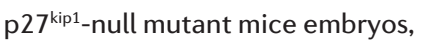
there is a marked decrease in neuronal production (due to fewer cells exiting the cell cycle). However, some neurons are produced, and Guillemot's team were interested in discovering what happens to them so that they could find out whether p $27^{\mathrm{kip} 1}$ might be involved in

This multitalented protein performs each step by a different mechanism.

additional developmental tasks. They labelled and followed the fate of the newly born neurons and observed that proportionately fewer cells migrated to the cortical plate from their birth place in the ventricular zone and that fewer cells expressed HuC/D protein, a marker of post-mitotic neurons. These results indicate that both migration and differentiation were impaired in the absence of $\mathrm{p} 27^{\mathrm{kip} 1}$. By overexpressing $\mathrm{p} 27^{\mathrm{kip} 1}$ in the mutant mice, both defects were rescued.

In p27ck embryos, as in p27 $7^{\text {kip } 1}$ null embryos, a decrease in neuronal production is observed ( $\mathrm{p} 27^{\mathrm{ck}}$ mice express a version of the $\mathrm{p} 27^{\mathrm{kip} 1}$ protein that fails to promote cell cycle exit). However, unlike p $27^{\mathrm{kip} 1}$-null embryos, the neurons that are produced in $\mathrm{p} 27^{\mathrm{ck}}$ embryos migrate and differentiate normally. Furthermore, the overexpression of $\mathrm{p} 27^{\mathrm{ck}}$ in $\mathrm{p} 27^{\mathrm{kip} 1}-\mathrm{knockdown}$ embryos rescues both the migration and differentiation defects, suggesting that the regulation of these processes by p $27^{\text {kip } 1}$ involves an independent mechanism from that involved in cell cycle regulation.

Guillemot and colleagues suspected that this new mechanism might involve neurogenin 2 ( $\mathrm{Ngn} 2$ ), as it too has been shown to regulate neuronal differentiation and migration. However, when Ngn2 was overexpressed in p27 $7^{\text {kip } 1-k n o c k d o w n ~}$ mice the differentiation defect was rescued but the migration defect was not. Instead, the migration defect was found to be rescued by overexpression of a dominant-negative form of RhoA - inactivation of RhoA had previously been shown to be necessary for $\mathrm{p} 27^{\mathrm{kip} 1}$-induced fibroblast migration.

To complete the study, the authors went on to determine the different parts of the $\mathrm{p} 27^{\mathrm{kip} 1}$ protein responsible for its two newly discovered functions. Altogether, the work by Guillemot's group provides extensive insight into both the workings of this multi-talented protein and the mechanisms underlying neurogenesis.

Ruth Williams

ORIGINAL RESEARCH PAPER Nguyen, L. et al. p $27^{\text {kip } 1} 1$ independently promotes neuronal differentiation and migration in the cerebral cortex. Genes Dev. 16 May 2006 (doi:10.1101/gad.377106) 


\section{Same but different}

GABA ( $\gamma$-aminobutyric acid), the main inhibitory neurotransmitter in the vertebrate nervous system, has many modes of action. For one of its receptors $\mathrm{GABA}_{\mathrm{B}}-\mathrm{a}$ heterodimer consisting of $\mathrm{GABA}_{\mathrm{B} 1}$ and $\mathrm{GABA}_{\mathrm{B} 2}$ subunits - functional diversity is achieved by different synaptic localization and physiological action of its subunit isoforms $\mathrm{GABA}_{\mathrm{B} 1 \mathrm{a}}$ and $\mathrm{GABA}_{\mathrm{B} 1 \mathrm{~b}}$, according to two studies published in Neuron.

To uncover the role of different $\mathrm{GABA}_{\mathrm{B} 1}$ versions in inhibitory neurotransmission, Bernhard Bettlers and colleagues used an ingenious approach to generate knockout mice in which $\mathrm{GABA}_{\mathrm{B} 1 \mathrm{a}}$ and $\mathrm{GABA}_{\mathrm{B} 1 \mathrm{~b}}$ were inactivated one at a time. Electron microscopy studies showed that, in the hippocampus, $\mathrm{GABA}_{\mathrm{Bla}}$ was predominantly localized at glutamatergic terminals, whereas $\mathrm{GABA}_{\mathrm{B} 1 \mathrm{~b}}$ was mainly found at dendritic spines opposite to the glutamate release sites. This differential localization of $\mathrm{GABA}_{\mathrm{B} 1}$ isoforms correlated with their functional differences: at hippocampal CA3-to-CA1 synapses, $\mathrm{GABA}_{\mathrm{Bla}}$ assembled receptors that blocked presynaptic glutamate release, while $\mathrm{GABA}_{\mathrm{B} 1 \mathrm{~b}}$ was involved in postsynaptic inhibition of neuronal firing. Intriguingly, the constitutive absence of $\mathrm{GABA}_{\mathrm{B} 1 \mathrm{a}}$, but not $\mathrm{GABA}_{\mathrm{B} 1 \mathrm{~b}}$, resulted in impaired synaptic plasticity and hippocampus-dependent memory formation. This suggests that different $\mathrm{GABA}_{\mathrm{B}}$ receptor compositions might mediate distinct neural functions.
In a companion paper, PerezGarci and colleagues report similar results of distinct $\mathrm{GABA}_{\mathrm{B} 1}$ receptor functions in pyramidal neurons residing in layer 5 (L5) of the neocortex. These L5 neurons are innervated by numerous inhibitory inputs, including those from interneurons in L1. The researchers first established that extracellular stimulation of L1 inhibited the dendritic $\mathrm{Ca}^{2+}$ spikes in L5 pyramidal neurons. Further, the short-lasting component of this inhibitory effect was mediated by GABA receptors, whereas the long-term counterpart was mediated by $\mathrm{GABA}_{\mathrm{B}}$ receptors. Using the knockout mice generated by Bettler et al., the researchers found that L5 pyramidal neurons from $\mathrm{GABA}_{\mathrm{B} 1 \mathrm{a}}$-deficient mice showed normal short- and longlasting inhibitory components in response to $\mathrm{L} 1$ stimulation. By contrast, the absence of $\mathrm{GABA}_{\mathrm{B} 1 \mathrm{~b}}$ completely abolished the long-term inhibitory response of L5 pyramidal neurons, but the $\mathrm{GABA}_{\mathrm{A}}$-mediated

\section{Dendritic spines go retro}

Endocannabinoids are unconventional neurotransmitters, travelling from a postsynaptic to a presynaptic neuron - the opposite direction to typical chemical messengers. This signalling is known to be important for the survival, differentiation and function of the presynaptic neuron, but there is speculation about the synaptic site of production of endocannabinoids and how they elicit their responses. Katona and colleagues go some way to clarifying the molecular architecture of the endocannabinoid system at glutamatergic synapses, and also implicate this pathway in the plasticity of these synapses.

Thought to be produced postsynaptically in response to specific physiological stimuli, endocannabinoids have several potential cellular targets, two of which are the cannabinoid receptors $\mathrm{CB}_{1}$ and $\mathrm{CB}_{2}$.
Of these receptors, so far only $\mathrm{CB}_{1}$ has been found at the presynapse. If endocannabinoids act on $\mathrm{CB}_{1}$ presynaptically, as has been proposed, these molecules are prime candidates as retrograde signals in synaptic plasticity. In the hippocampus, the predominant endocannabinoid thought to be involved in synaptic plasticity is the fatty acid derivative 2-arachidonoyl-glycerol (2-AG), the synthesis of which is thought to be mediated by the enzyme diacylglycerol lipase- $\alpha$ (DGL $\alpha$ ). The authors found that the highest expression levels of DGL $\alpha$ were indeed in the hippocampus, and in particular at the heads of dendritic spines - sites of synaptic plasticity for glutamatergic synapses - of hippocampal principal cells.

Dendritic spines are made up of discrete functional subunits that individually contribute to separate aspects of synaptic plasticity. Could the site of DGL $\alpha$ expression be narrowed even further? High-resolution immunoelectron microscopy revealed that DGL $\alpha$ production at glutamatergic synapses is highest in the perisynaptic annulus that surrounds the postsynaptic density, with the active site of DGL $\alpha$ being located on the intracellular side of the membrane. Interestingly, the authors noted that the levels of DGL $\alpha$ expression decreased gradually along the spine neck. This finding lends support to the theory that the narrow spine acts as a barrier to ensure synapse-specific plasticity mechanisms.

... endocannabinoids... are prime candidates as retrograde signals in synaptic plasticity.

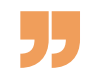

The localization of DGL $\alpha$ on dendritic spine heads suggests that the receptors for $2-\mathrm{AG}$ - that is, $\mathrm{CB}_{1}$

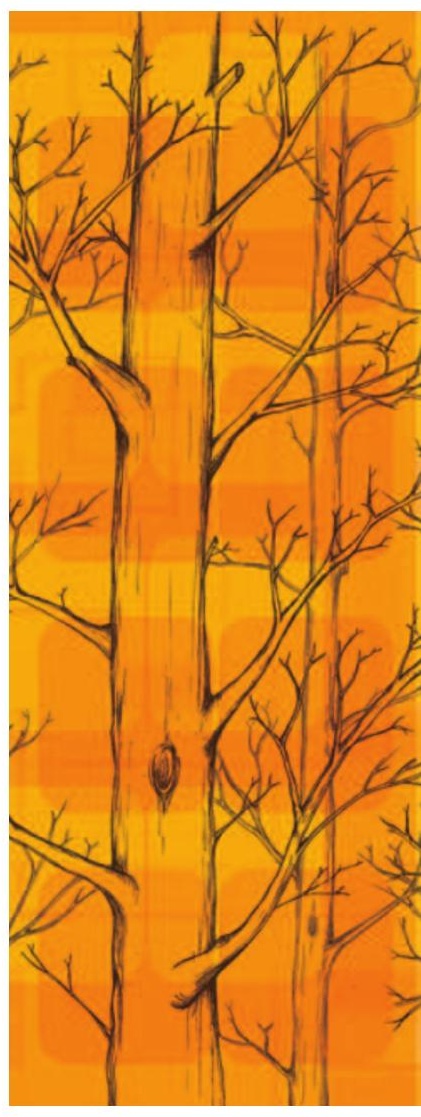


short-term inhibition was unaffected.

These two studies shed fresh light on the apparent contradiction between functional diversity and molecular simplicity of the $\mathrm{GABA}_{\mathrm{B}}$ receptor system. It will be interesting to test whether other GABA receptors deploy similar strategies to generate distinct activity under different physiological conditions.

Jane Qiu

ORIGINAL RESEARCH PAPERS Vigot, R. et al. Differential compartmentalization and distinct functions of $\mathrm{GABA}_{\mathrm{B}}$ receptor vaiants. Neuron $\mathbf{5 0}$, 589-601 (2006) | Perez-Garci, E. et al. The $\mathrm{GABA}_{\mathrm{B} 1 \mathrm{~b}}$ isoform mediates long-lasting inhibition of dendritic $\mathrm{Ca}^{2+}$ spikes in layer 5 somatosensory pyramidal neurons. Neuron $\mathbf{5 0}$ 603-616 (2006)

FURTHER READING Farrant, M. \& Nusser, Z. Variations on an inhibitory theme: phasic and tonic activation of GABA receptors. Nature Rev. Neurosci. 6, 215-229 (2005) WEB SITES

Bettler's laboratory:

http://www.pharmazentrum.unibas.ch/bettler. html

Larkum's laboratory:

http://pylwww.unibe.ch/ larkum/

receptors - should be located in close proximity on the opposite side of the synapse. Indeed, immunocytochemical analyses revealed the localization of $\mathrm{CB}_{1}$ receptors to these specific axon terminals in wild-type mice but not in $\mathrm{CB}_{1}$-knockout mice. This precise positioning implicates 2-AG in retrograde signalling at glutamatergic synapses.

On the basis of these findings, the authors propose that $\mathrm{CB}_{1}$ receptors could control glutamate release presynaptically, and so be involved in homosynaptic plasticity of excitatory synapses and heterosynaptic plasticity between excitatory and inhibitory contacts. It will be interesting to see whether other biosynthetic routes to 2-AG exist at other synapses and, if they do, whether their molecular architecture matches that of glutamatergic synapses.

Samantha Barton

ORIGINAL RESEARCH PAPER Katona, l. et al. Molecular composition of the endocannabinoid system at glutamatergic synapses. J. Neurosci. 26 , 5628-5637 (2006)

NEUROLOGICAL DISORDERS

\section{Changing channels}

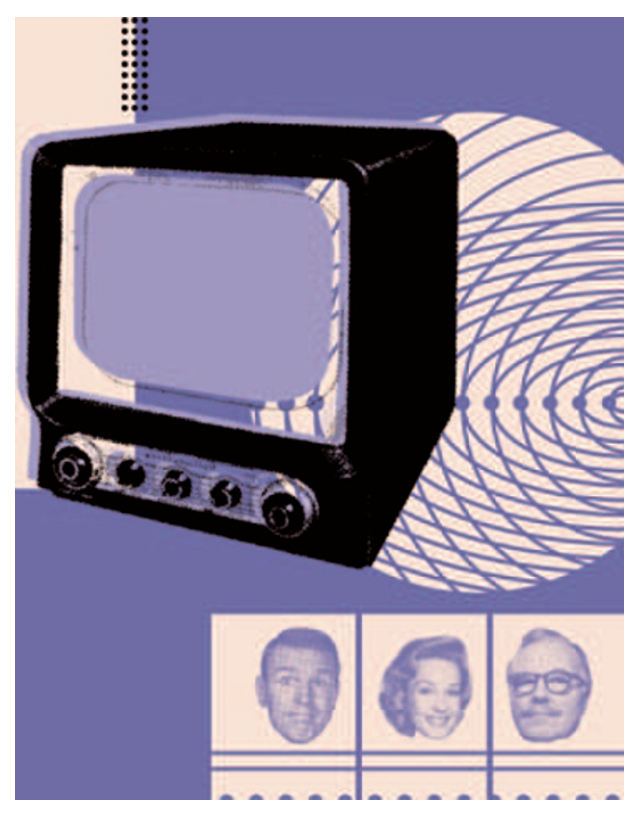

Ischaemic injury involves a pronounced reduction in oxygen and glucose around the affected area, which leads to rapid cell death that is associated with $\mathrm{Ca}^{2+}, \mathrm{Na}^{+}, \mathrm{K}^{+}$and $\mathrm{Cl}^{-}$dysregulation. But which channels mediate these changes? Writing in Science, Thompson and colleagues report the surprising finding that the ionic dysregulation seen in ischaemia results from the opening of hemichannels - sets of proteins that form one half of large conductance channels, known as gap junctions, that allow molecules and ions to flow directly between most cell types.

Thompson and co-workers created ischaemiclike conditions in which there was oxygen and glucose deprivation (OGD) in isolated rat hippocampal pyramidal neurons. Voltage-clamp recordings revealed that OGD activated a current with a large amplitude, a linear current-voltage relationship and a reversal potential close to $0 \mathrm{mV}$ - key features of hemichannel activation when $\mathrm{Na}^{+}, \mathrm{Ca}^{2+}$ and $\mathrm{K}^{+}$currents were blocked. Selective blockage of hemichannels using carbenoxolone (Cbx) or lanthanum chloride confirmed their involvement in OGD-activated current. Prolonged (>20 min) OGD led to irreversible current activation, neuronal swelling and breakdown of cell membranes.

Further investigations showed that blocking the ion channel ASIC1a or the purinergic $\mathrm{P} 2 \mathrm{X}_{7}$ receptor (previously implicated in ischaemiarelated ionic dysregulation) failed to affect the large current triggered by OGD activation, although there was a minor effect of blocking the transient receptor potential channels TRPC4 and TRPC 5 on the small residual current that remained after blockage of hemichannels. Nevertheless, the large amplitude current attributable to hemichannels indicated that they are the major contributors to the OGD-activated current, so these researchers explored their role in ionic dysregulation in more detail.

\section{Hemichannels represent a promising therapeutic target in the treatment of ischaemic injury.}

Thompson and colleagues separately assessed the movement of two types of small fluorescent molecule that can permeate gap junctions: calcein AM and sulphorhodamine (SR101). Under normal conditions calcein AM remains inside neurons, whereas SR101 is absent from neurons. But during OGD there was a steady reduction in calcein and an increase in SR101 in neurons. The ability of these molecules to cross the membrane was prevented by $\mathrm{Cbx}$, indicating that hemichannel opening was responsible for their movement.

Gap junctions are formed from two hemichannels that comprise connexin or pannexin (PX) proteins. Pannexins, but not connexins, are expressed in pyramidal neurons and were therefore likely to form the hemichannels under scrutiny here. The singlechannel biophysical properties - including amplitude, conductance, opening probability, current-voltage relationship and reversal potential - were consistent with those of PX1 hemichannels.

This comprehensive set of studies provides compelling evidence that the opening of PX1 gap junction hemichannels is crucial for the ionic dysregulation and consequent neuronal death that follows ischaemia. Hemichannels therefore represent a promising therapeutic target in the treatment of ischaemic injury.

Alison Rowan

ORIGINAL RESEARCH PAPER Thompson, R. J., Zhou, N. \& MacVicar, B. A. Ischemia opens neuronal gap junction hemichannels. Science 312, 924-927 (2006) FURTHER READING Sohl, G., Maxeiner, S. \& Willecke, K. Expression and functions of neuronal gap junctions. Nature Rev. Neurosci. 6, 191-200 (2005) 


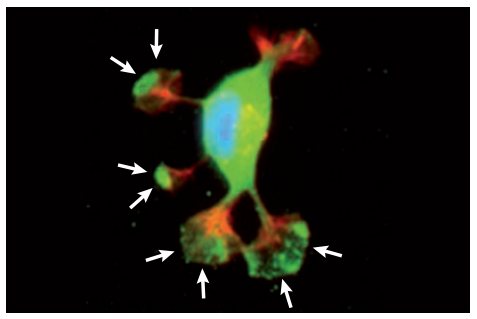

Fluorescence image of WAVE1 localization in lamellipodia of an early-stage oligodendrocyte purified from neonatal rat forebrain. WAVE1 (green) staining localizes to lamellipodia (arrows) of an oligodendrocyte. Red staining represents F-actin. Image courtesy of T. Vartanian and J. A. Sloane, Harvard Medical School, Massachusetts, USA.

G L I A

\section{Making waves for myelin}

The outgrowth of oligodendrocyte cell processes is vital for the formation of myelin, but the underlying mechanisms are not well understood. Kim, DiBernardo and colleagues now take us a step closer to understanding these mechanisms with the discovery that a WAVE (Wiskott-Aldrich syndrome protein family verprolin homologous) protein is essential for the morphological development of oligodendrocytes and consequent myelin formation through its influence on cytoskeleton dynamics.

WAVE proteins are widely expressed in the nervous system, but little is known about their functions, particularly in glia. One clear role for these proteins in neurons, however, is that they are involved in regulating the organization of the actin cytoskeleton - which is also a crucial step in the generation of myelin sheaths.

These researchers found that the WAVE1 isoform, but not WAVE2 or WAVE3, is dynamically expressed in oligodendrocytes during development. Moreover, WAVE1 expression coincided with the onset of myelination, and was localized to the leading edge of lamellipodia - actin cytoskeleton projections that reside on migrating cells. The team therefore investigated the function of WAVE1 in oligodendrocytes.

Preventing WAVE1 function disrupted process extension in cultured oligodendrocytes and in oligodendrocytes from WAVE1-knockout mice. However, although this isoform is also expressed in neurons, their development was unaffected. Furthermore, in WAVE1-knockout mice, formation of nodes of Ranvier was compromised, suggesting that WAVE1 is crucial for normal myelination.

These abnormalities in oligodendrocyte development had a profound effect on the overall anatomy of the brain in WAVE1knockout mice. There was a general reduction in brain size, with volume loss being most pronounced in the forebrain and corpus callosum, whereas the lateral ventricles were significantly larger than normal. These findings were associated with an overall reduction in the number of oligodendrocytes participating in myelination, particularly in the corpus callosum.

This elegant study not only sheds light on the mechanisms of myelin formation, but also reveals an important role for WAVE1 in glia. It will be interesting to determine whether WAVE1 contributes to oligodendrocyte maturation as well as the development of myelin.

Alison Rowan

ORIGINAL RESEARCH PAPER Kim, H.-J., DiBernardo, A. B. et al. WAVE1 is required for oligodendrocyte morphogenesis and normal CNS myelination. J. Neurosci. 26 , 5849-5859 (2006)

FURTHER READING Sherman, D. L. \& Brophy, P.J. Mechanisms of axon ensheathment and myelin growth. Nature Rev. Neurosci. 6, 683-690 (2005)

\section{Extremes of excitability}

Mutations in ion channels, usually characterized as producing either hyperexcitability or hypoexcitability, are associated with various neurological disorders. Now, Stephen Waxman and colleagues report that the same mutation in the sodium channel Nav1.7 has opposing effects on excitability in dorsal root ganglion (DRG) neurons and sympathetic ganglion neurons.

Nav1.7 is expressed mainly in primary sensory neurons such as DRG neurons and sympathetic ganglion neurons such as superior cervical ganglion (SCG) neurons, each of which possesses a different suite of sodium channel isoforms. The Nav1.7 mutations characterized so far produce changes in channel physiology that augment the response of the channel to small stimuli, but the effects of cell type on these changes had not been proposed that the same mutation

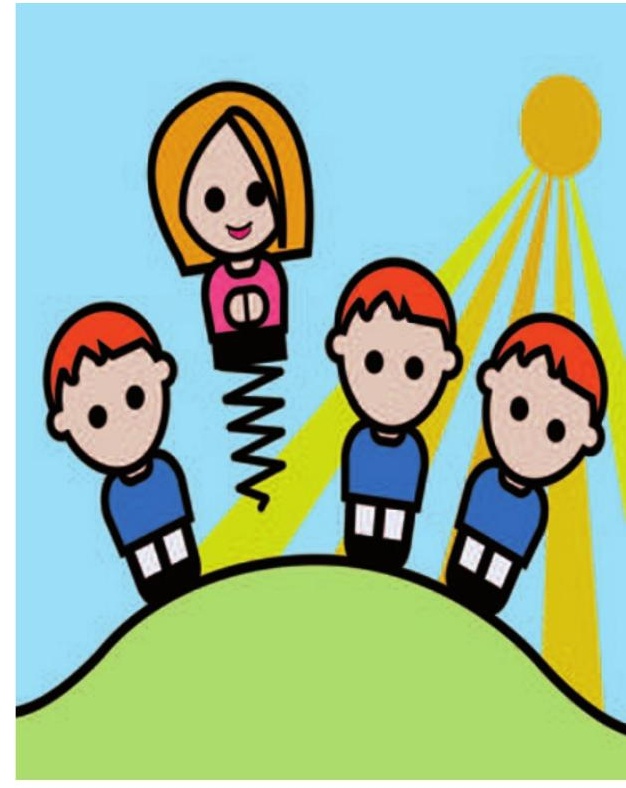

assessed. Waxman and colleagues might have different effects on

\section{NEUROIMAGING}

\section{Peering into the root of prejudice}

The human brain has the unique ability to represent the mental states of the self and others, and to make inferences about the relationship between them. Writing in Neuron, Jason Mitchell and colleagues report that different regions of the brain are active when we are thinking about people either politically alike or different from us.

According to the 'simulation' (or 'projection') theory of social cognition, we can predict what someone else will think and feel by considering what we would think and feel if we were in their situation. However, if the target of prediction is substantially different from us, using self-knowledge to inform such inferences might be less useful. The researchers conjectured that there might be a refined 'division of labour'<smiles>C1CC2CC1CC1CC2C1</smiles>

... different regions of the brain are active when we are thinking about people either politically alike or different from us.

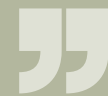

in the contributions by different subregions of the medial prefrontal cortex (mPFC) - the primary region of the brain implicated in social cognition - to making inferences about the mental states of others.

To detect the possible 'imprinting' of similarity and dissimilarity on the 
excitability in different neurons, and that the ensemble of channels present in a cell might be an important determinant of these effects.

To test this hypothesis, the authors studied the functional effects of the Nav1.7 mutation $\mathrm{L} 858 \mathrm{H}$, which is associated with the neuropathic pain syndrome erythermalgia, in cultured DRG and SCG neurons. They showed that the mutation depolarized the resting membrane potential (by $\sim 5 \mathrm{mV}$ relative to wild-type Nav1.7) in both types of neuron. However, it led to opposite changes in the current threshold for firing a single action potential, which was decreased in DRG neurons but increased in SCG neurons. The firing frequency of neurons in response to prolonged stimulation was also altered in an opposing manner, being lower in DRG neurons but higher in SCG neurons.

Action potential generation in DRG neurons involves the sequential activation of Nav1.7 channels followed by Nav1.8 channels, which are relatively resistant to inactivation by depolarization. To determine the role of other sodium channel isoforms in the apparently disparate effects of Nav1.7 mutation, the authors looked for differences in the isoforms expressed by both types of neuron. Immunocytochemistry of cultured cells and adult rat neurons revealed the presence of Nav1.7 protein in both types of neuron, and the presence of Nav1.8 protein in DRG neurons, but not SCG neurons. Coexpression of Nav1.8 and Nav1.7/ L858H in SCG neurons restored the action potential threshold and amplitude to near wild-type levels, despite the persistence of membrane depolarization.

These findings show that opposing functional effects can be induced by the same ion channel mutation, and provide a molecular basis for the sympathetic dysfunction observed in erythermalgia.

Daniel McGowan

ORIGINAL RESEARCH PAPER Rush, A. M. et al. A single sodium channel mutation produces hyper- or hypoexcitability in different types of neurons. Proc. Natl Acad. Sci. USA 103, 8245-8250 (2006)

students were mentalizing a target with similar political views, whereas the dorsal mPFC region was more active when the students were considering a target with views on the different end of the political spectrum from their own.

This elegant study has provided strong evidence for segregation of function within the area of mPFC associated with predicting the mental states of the self and of others. Whether this division of labour underlies the neural mechanisms of prejudice remains to be seen. Jane Qiu than their Hollywood counterparts. Finally, the students completed a version of the Implicit Association Test, which was designed to index how strongly they automatically associate themselves with the liberal or conservative target.

As the researchers predicted, the brain showed different activation patterns when the students were thinking about a politically similar or dissimilar target. The ventral mPFC region was more engaged when the
ORIGINAL RESEARCH PAPER Mitchell, J. P. et al. Dissociable medial prefrontal contributions to judgments of similar and dissimilar others. Neuron 50, 655-663 (2006) FURTHER READING Amodio, D. M. \& Frith, C. D. Meeting of minds: the medial frontal cortex and social cognition. Nature Rev. Neurosci. 7, 268-277 (2006) | Haynes, J.-D. \& Rees, G. Decoding mental states from brain activity in humans. Nature Rev. Neurosci. 7, 523-534 (2006)

WEB SITE

Banaji's laboratory:

http://www.people.fas.harvard.edu/ banaji/

\section{IN BRIEF}

\section{$\Rightarrow$ SYNAPTIC PHYSIOLOGY}

Miniature neurotransmission stabilizes synaptic function via tonic suppression of local dendritic protein synthesis.

Sutton, M. A. et al. Cell 125, 785-799 (2006)

Long-term blockade of neuronal activity induces compensatory increases in neurotransmitter release. Miniature synaptic events ('minis') are thought to be crucial for this process, but their precise function is not known. Sutton et al. show that blockade of NMDA receptor-mediated 'minis' rapidly scales up the amplitude of AMPA miniature excitatory postsynaptic currents, which requires local protein synthesis and the insertion of synaptic AMPA receptors. The results suggest that NMDA signalling during miniature synaptic transmission serves to stabilize synaptic function through active suppression of dendritic protein synthesis.

\section{PAIN}

Prociceptive and antinociceptive effects of estradial through endogenous opioid neurotransmission in women.

Smith, Y. R. et al. J. Neurosci. 26, 5777-5785 (2006)

Smith et al. provide functional evidence on the role of oestrogen in the regulation of the $\mu$-opioid receptor. The researchers recruited women in the early follicular phase of their menstrual cycle, during which the level of oestrogen is low, and used positron emission tomography to examine their pain responses before and after oestradiol treatment. In regions of the brain associated with sex-dependent differences in pain processing, oestradiol was shown to increase the basal level of $\mu$-opioid receptors and enhance the activation of opioid neurotransmission.

\section{$\Rightarrow$ NEURODEGENERATIVE DISORDERS}

Onset and progression in inherited ALS determined by motor neurons and microglia.

Boillée, S. et al. Science 312, 1389-1392 (2006)

Mutations in superoxide dismutase (SOD1) result in amyotrophic lateral sclerosis (ALS). Boillee et al. now show that the initiation and progression of ALS are distinct phases of the disease. Expression of mutant SOD1 in motor neurons was found to be a key factor in the initiation, but not the progression, of ALS-like disease in mice, whereas its expression in microglia was the primary determinant of disease progression.

\section{$\Rightarrow$ BEHAVIOURAL NEUROSCIENCE}

Cortical substrates for exploratory decisions in humans.

Daw, N. D. et al. Nature 15 June 2006 (doi:10. 1038/nature04766)

In an uncertain environment, we can either choose familiar options with known rewards (exploitative behaviour) or unfamiliar options with riskier, but potentially more advantageous, outcomes (exploratory behaviour). Daw et al. used a gambling task designed to tease apart these two types of behaviour during functional MRI studies. Exploratory decisions were associated with activation of the frontal pole of the prefrontal cortex, whereas the striatum and ventromedial prefrontal regions were active during exploitative decision making. The authors therefore propose that decision making in uncertain situations entails switching between exploitative and exploratory behaviour. 